\title{
Densities of states and the Cohen-Kaplan-Nelson bound
}

\author{
Nikita Blinov $\oplus^{1,2}$ and Patrick Draper $\oplus^{3}$ \\ ${ }^{1}$ Fermi National Accelerator Laboratory, Batavia, Illinois 60510, USA \\ ${ }^{2}$ Kavli Institute for Cosmological Physics, University of Chicago, Chicago, Illinois, USA \\ ${ }^{3}$ Illinois Center for Advanced Studies of the Universe \& Department of Physics, University of Illinois, \\ Urbana, Illinois 61801, USA
}

(Received 25 July 2021; accepted 1 October 2021; published 26 October 2021)

\begin{abstract}
The holographic principle implies that quantum field theory (QFT) overcounts the number of independent degrees of freedom in quantum gravity. An argument due to Cohen, Kaplan, and Nelson (CKN) suggests that the number of degrees of freedom well described by QFT is even smaller than required by holographic bounds, and CKN interpreted this result as indicative of a correlation between the UV and IR cutoffs on QFT. Here, we consider an alternative interpretation in which the QFT degrees of freedom are depleted as a function of scale. We use a simple recipe to estimate the impact of depleted densities of states on precision observables, including the Lamb shift and lepton $g-2$. Although these observables are not sensitive to the level of depletion motivated by gravitational considerations, the phenomenological exercises also provide an interesting test of quantum field theory that is independent of underlying quantum gravity assumptions. A depleted density of states can also render the QFT vacuum energy UV insensitive, reconciling the success of QFT in describing ordinary particle physics processes and its apparent failure in predicting the cosmological constant.
\end{abstract}

DOI: 10.1103/PhysRevD.104.076024

\section{INTRODUCTION}

We do not know how realistic quantum field theories emerge as a low-energy approximation to a complete theory of quantum gravity with a positive cosmological constant (cc). However, it is likely that quantum field theory (QFT) contains too many degrees of freedom, even with an ultraviolet cutoff $\Lambda$ well below the Planck scale. In QFT, the maximum entropy localized in a box of size $L$ scales extensively as $S \sim(\Lambda L)^{3}$, while the holographic principle limits the number of degrees of freedom in quantum gravity to $S \sim M_{p}^{2} L^{2}$. Furthermore, this overabundance of QFT states does not mean that most of the states in quantum gravity have to behave like bulk QFT states. A hot box containing QFT degrees of freedom collapses to a black hole when $T^{4} L^{3} / M_{p}^{2} \sim L$, corresponding to an entropy $S \sim\left(M_{p} L\right)^{3 / 2} \ll M_{p}^{2} L^{2}$. It could well be that the number of degrees of freedom in the correct quantum theory of gravity that are well described by bulk quantum field theory is only of order $L^{3 / 2}[1,2]$.

Let us first recall some old ideas for how bulk particles might realize holographic bounds on degrees of freedom

Published by the American Physical Society under the terms of the Creative Commons Attribution 4.0 International license. Further distribution of this work must maintain attribution to the author(s) and the published article's title, journal citation, and DOI. Funded by SCOAP ${ }^{3}$.
[3-6]. In Ref. [6], particles are modeled as a collection of indivisible partons in light front quantization. The number of partons one should ascribe to a particle grows with the laboratory frame energy $\epsilon$ and the resolution with which its momentum can be measured: $N_{\text {parton }} \sim \epsilon L$, where we describe the momentum resolution as an infrared length scale, $\Delta p=1 / L$. Once the number of partons exceeds some inverse coupling of the microscopic quantum gravity theory, for example the string coupling $g_{s}^{2}$, the size of the particle $R$ begins to grow rapidly with each additional parton. It scales as $M_{p} R \sim(\epsilon L)^{1 /(d-1)}$ in $d$ spatial dimensions, so that $M_{p} R \sim \sqrt{\epsilon L}$ in $d=3$.

When the particle size is so large that it can no longer be localized on length scales of order the inverse energy, it ceases to be pointlike and cannot be regarded as an excitation of one independent degree of freedom. However, in the parton model, this limit depends on the momentum resolution. We could interpret the saturation of $R=1 / \epsilon$ as supplying a bound on the typical momentum spacing between individual particle degrees of freedom around $\epsilon$,

$$
1 / L_{\min }=\epsilon^{3} / M_{p}^{2}
$$

The spacing grows with energy, rapidly depleting the single-particle density of states (DOS) $g(\epsilon)$. In a box of fixed size $L$, there is an energy scale $\hat{e}=\left(M_{p}^{2} / L\right)^{1 / 3}$ at 
which the momentum resolution transitions from $1 / L$ to $1 / L_{\min }$. Here, the relativistic DOS transitions from ordinary QFT scaling $g \sim L^{3} \epsilon^{2}$ to $g \sim L_{\min }^{3} \epsilon^{2}$. Most of the degrees of freedom live around $\hat{\epsilon}$, and the maximum entropy is of order $(L \hat{\epsilon})^{3} \sim\left(L M_{p}\right)^{2}$, satisfying the holographic bound. Alternatively, the same result is obtained by counting states according to the scaling $g \sim \partial_{\epsilon}\left(L^{3} / R^{3}\right)$ for $R>1 / \epsilon$.

We see that the partonic model realizes the holographic principle by a scale-dependent depletion of the independent degrees of freedom, relative to the density of states of an ordinary bulk field theory in a fixed volume.

However, as alluded to above, there is an argument due to Cohen, Kaplan, and Nelson $(\mathrm{CKN})$ that the number of degrees of freedom that can be well described by bulk QFT might be even smaller than what is implied by holography [1]. CKN interpreted the entropy scaling $S \sim\left(M_{p} L\right)^{3 / 2}$ as implying a correlation between the UV and IR cutoffs $\Lambda$ and $L$ on QFT: requiring $(\Lambda L)^{3} \lesssim\left(M_{p} L\right)^{3 / 2}$, one arrives at the bound

$$
L \lesssim M_{p} / \Lambda^{2} .
$$

For a weak-scale UV cutoff, $L$ is the order of $1 \mathrm{~cm}$ when the bound is saturated. What this IR scale means, however, is unclear. In Ref. [1], it was interpreted as an IR momentum cutoff on Feynman graphs for a given UV cutoff, and recently, there has been renewed interest in this interpretation of the bound and its possible implications for precision measurements [7-9]. In fact, this interpretation suggests that the effects of quantum gravity on precision observables are so large that they may be detectable following plausible experimental improvements [1,7-9].

In this paper, we consider a different interpretation, introduced in Ref. [10], where $1 / L$ is taken to be a bound on a characteristic momentum spacing between independent degrees of freedom that can be well described by bulk quantum field theory. In other words, $L$ controls a depletion of the single-particle density of states of ordinary QFT, and the depletion may be stronger than what is required by holography alone.

An insightful observation made in Refs. [1,11] was phrased in Ref. [1] as follows: "There is in fact no evidence that fields at present experimental energies can fluctuate independently over a region as large as our horizon." Over how large a region can localized excitations of a typical energy fluctuate independently? This is both an empirical question and a question of principle. In order to localize an excitation of energy approximately $\epsilon$ in, say, a minimal region of size $1 / \epsilon$, inside of a much larger region approximately $L$, there must be many independent modes around $\epsilon$ with momentum spacing $\Delta p \sim 1 / L$. Since no real experiment is sensitive to arbitrarily small differences in momenta or energy, however, there are only empirical lower bounds on the QFT single-particle density of states. As a matter of principle, we interpret the bound (2) as a fundamental upper limit on the DOS. The bound (2) suggests that the relativistic DOS is depleted above some energy scale, behaving as

$$
g \sim\left\{\begin{array}{ll}
L^{3} \epsilon^{2} & \epsilon^{2} \ll M_{p} / L \\
M_{p}^{3} / \epsilon^{4} & \epsilon^{2} \gg M_{p} / L
\end{array} .\right.
$$

We can parametrize the scale-dependent depletion in terms of an effective box size at different energy scales, $L_{\text {eff }}(\epsilon)=\min \left(L, M_{p} / \epsilon^{2}\right)$. This interpretation is similar to the holographic partonic picture described above, but with different scalings, such that $L_{\text {eff }}$ of Eq. (3) is smaller than the holographic $L_{\min }$ of Eq. (1).

In this work, we will consider first the empirical question: how well has the single-particle density of states been tested at various energy scales? This assessment is independent of any more fundamental motivations about quantum gravity and represents an interesting, somewhat unconventional axis along which quantum field theory can be tested. Simple estimates can be made based on the sensitivity of detectors, while less trivial estimates arise from precision measurements of observables that involve sums over intermediate states at characteristic energies $\epsilon$. In the latter category, we consider atomic spectra and leptonic $g-2$, using finite-volume techniques to impose a discretization of the state space. In this way, we can associate an empirical lower bound on the IR scale $L_{\text {eff }}(\epsilon)$ from these measurements. We compare the empirical bounds on $L_{\text {eff }}$ to (3), finding that current precision falls well short of the modifications suggested by these gravitational arguments. Thus, this is an interpretation of the CKN bound that does minimal violence to the predictions of ordinary QFT.

We then reexamine the CKN "hot box" thought experiments using the depleted DOS in Eq. (3) and generalize it to other power laws. We note that there are some nonthermal, high-occupancy states which would collapse to a black hole in a gravitational theory and which are not removed either by depleting the single-particle DOS or by placing correlated cutoffs on Feynman integrals. Some states must be excised by other effects.

We emphasize that we do not have any concrete proposal for how a fundamental depletion of QFT modes should be implemented nor do we know how Lorentz invariance and locality should emerge. In Matrix Theory, a theory which realizes the holographic principle yet gives rise to a supergravity theory at long distances, Lorentz invariance and locality of scattering amplitudes are thought to result from delicate cancellations and depend intricately on the Bogomol'nyi-Prasad-Sommerfield nature of the partons [12]. We will at least not assume any modification to the relativistic dispersion relations, which provide the most sensitive experimental probes of Lorentz invariance, and our results for $g-2$ and the Lamb shift differ from the predictions of ordinary relativistic QFT by $\mathcal{O}\left(1 / M_{p}^{2}\right)$. 
These exercises have curious implications for the cosmological constant problem. The QFT contribution to the total vacuum energy can be written as

$$
\Delta \rho \sim V^{-1} \int \tilde{g}(\epsilon) \epsilon d \epsilon,
$$

which is quartically divergent for the usual DOS. This expression depends on the graded DOS $\tilde{g}=(-1)^{F} g$ where $F=0$ (1) for bosons (fermions). Exact supersymmetry solves the cosmological constant problem within quantum field theory alone because it depletes the graded DOS completely, leaving unaffected the ordinary DOS for different species. A few other distinct and fascinating examples in quantum field theory also yield cancellations in the graded DOS $[13,14]$. We are instead considering the possibility that the DOS is depleted for each species separately and that this depletion is intrinsically gravitational-it disappears in the $M_{p} \rightarrow \infty$ limit. With the depletion (3), for example, the fine-tuning problem is removed. Moreover, we will conclude that the bounds on the DOS obtained from precision particle physics measurements are far from definitively establishing that the cc problem is a problem, without invoking a substantial extrapolation.

\section{EMPIRICAL PROBES OF THE QFT DENSITY OF STATES}

The density of states enters into the sum over intermediate states in precision processes. For such processes, we can estimate the sensitivity to the DOS using finite-volume techniques. Placing a theory in a periodic box of volume $L^{3}$, the DOS of a massless particle is depleted in a scaleindependent way from the continuum to $g \sim \epsilon^{2} L^{3}$. Standard techniques can be used to compute the $L$-dependent effects on the process, typically considered as a power series in $1 / L$ for large boxes. Since we are interested in the sensitivity of observables to the DOS at different scales, we proceed as follows. Given an observable $\mathcal{O}$ computed in perturbation theory, we isolate in some manner the perturbative corrections to $\mathcal{O}$ from intermediate states where the individual particles all have some characteristic energy $\epsilon_{*}$. Call these contributions $\mathcal{O}_{\epsilon_{*}}$. Placing the theory in a box of size $L$, we then compute the $1 / L$ corrections to $\mathcal{O}_{\epsilon_{*}}$, denoted $\mathcal{O}_{\epsilon_{*}}^{L}$. Comparing $\mathcal{O}_{\epsilon_{*}}^{L}$ to the experimental precision with which $\mathcal{O}$ is known, we can place a lower bound on the box size,
$L>L_{\mathcal{O}}$. We identify this bound as the current experimental limit on the depleted density of states at $\epsilon_{*}$, parametrized by the effective length scale $L_{\text {eff }}$ :

$$
L_{\text {eff }}\left(\epsilon_{*}\right)>L_{\mathcal{O}} .
$$

In other words, the impact of the depletion can be approximated as a finite-volume effect in a box of size $L_{\text {eff }}\left(\epsilon_{*}\right)$. Corrections to this estimate depend on $\Delta \epsilon / \epsilon_{*}$, where $\Delta \epsilon$ is the range of energies allowed to contribute to $\mathcal{O}_{\epsilon_{*}}$.

Such estimates can be performed entirely numerically. However, we will find it convenient to take a slightly different approach. We will compute the entire $L$-dependent effects on observables $\mathcal{O}$ analytically as a series in $1 / L$, then inspect the terms to see which intermediate energy scales contribute dominantly to each power of $1 / L$.

What these methods lack in sophistication, we hope they make up for in generality; since at best we obtain only conservative, order-of-magnitude bounds on $L_{\text {eff }}$, they might have some resilience against whatever detailed form the actual depletion takes.

We begin by computing the modification to the Coulomb potential and infer bounds on $L_{\text {eff }}\left(\epsilon_{\star}=\alpha m_{e}\right)$ from atomic physics. We then consider state depletion effects on $L_{\text {eff }}\left(\epsilon_{\star}=m_{e}\right)$ from $g-2$, since that precisely known quantity has been of particular interest in the CKN-related literature [1,7-10]. Finally, we briefly consider bounds at higher energies based on the measured resolution of detectors.

\section{A. Coulomb forces and bound state energies}

The depletion of single-particle states can affect the electromagnetic force in atomic physics. In standard QFT, the Coulomb potential is

$$
V(\mathbf{r})=-\int \frac{d^{3} q}{(2 \pi)^{3}} \frac{e^{2}}{\mathbf{q}^{2}} e^{i \mathbf{q} \cdot \mathbf{r}}=-\frac{e^{2}}{2 \pi^{2} r} \int_{0}^{\infty} d \tilde{q} \frac{\sin \tilde{q}}{\tilde{q}} .
$$

In old-fashioned perturbation theory, the integral over $q$ corresponds to a sum over on-shell intermediate states with momentum $\mathbf{q}$ and energy $E=|\mathbf{q}|$. We leave the final integral over $\tilde{q}=q r$ unevaluated in Eq. (6) to highlight the fact that the largest contribution comes from modes with $q r \lesssim 1$.

As described above, we model a depletion of the DOS by putting the system in a box of size $L$. Following Refs. [15,16], we rewrite the integrand in Eq. (6) in Schwinger parametrization and discretize Cartesian momenta $\mathbf{q}_{i} \rightarrow\left(2 \pi n_{i}\right) / L$ :

$$
\begin{aligned}
V(\mathbf{r}) & =-e^{2} \int_{0}^{\infty} d \rho \frac{1}{L^{3}} \prod_{i=1}^{3}\left\{\sum_{n_{i}=-\infty}^{+\infty} \exp \left(-\rho\left(\frac{2 \pi n_{i}}{L}\right)^{2}+i\left(\frac{2 \pi n_{i}}{L}\right) \mathbf{r}_{i}\right)\right\} \\
& =-\frac{e^{2}}{L^{3}} \int_{0}^{\infty} d \rho \prod_{i=1}^{3} \theta_{3}\left(-\frac{\pi \mathbf{r}_{i}}{L}, e^{-4 \pi^{2} \rho / L^{2}}\right)
\end{aligned}
$$


where

$$
\theta_{3}(z, \tau)=\sum_{n=-\infty}^{+\infty} \exp \left(i \pi n^{2} \tau+i 2 \pi n z\right)
$$

is the elliptic theta function. The integral in Eq. (7) is infrared divergent, which can be seen by expanding the integrand at $4 \pi^{2} \rho / L^{2} \gg 1$ :

$$
\begin{aligned}
& \prod_{i=1}^{3} \theta_{3}\left(-\frac{\pi \mathbf{r}_{i}}{L}, e^{-4 \pi^{2} \rho / L^{2}}\right) \\
& \quad \approx 1+2\left(\cos \frac{2 \pi x}{L}+\cos \frac{2 \pi y}{L}+\cos \frac{2 \pi z}{L}\right) e^{-4 \pi^{2} \rho / L^{2}}
\end{aligned}
$$

The constant term can be traced back to the zero modes in the first line of Eq. (7). This divergence is spurious and absent in the continuum where the integrand $d^{3} q / \mathbf{q}^{2}$ is finite as $\mathbf{q} \rightarrow \mathbf{0}$. It can be removed by subtracting the zero mode contribution [15],

$V_{R}(\mathbf{r})=-e^{2} \int_{0}^{\infty} d \rho \frac{1}{L^{3}}\left[\prod_{i=1}^{3} \theta_{3}\left(-\frac{\pi \mathbf{r}_{i}}{L}, e^{-4 \pi^{2} \rho / L^{2}}\right)-1\right]$

$$
\approx-\frac{e^{2}}{4 \pi r}\left(1-2.84 \frac{r}{L}+2.09 \frac{r^{3}}{L^{3}}+\ldots\right),
$$

where in the last line we chose $\mathbf{r}=r \hat{z}$ and expanded in $r / L{ }^{1}$ Even terms are absent in parentheses because $\theta_{3}$ is an even function of its first argument, and the numerical result in the final line is obtained by fitting a polynomial to $V_{R}(\mathbf{r}) /\left(-e^{2} /\right.$ $4 \pi r$ ). These coefficients can also be computed semianalytically by splitting the $\rho$ integration into a region with $\rho L^{2} \ll 1$ (where one can perform a small $\rho$ expansion but not an $r / L$ expansion) and another where one can expand in $r / L$; this calculation is presented in Appendix A. We have checked that these two approaches agree. For our purposes, all that is important is the powers of $r / L$. The $r \rightarrow 0$ limit of Eq. (11) also matches the results of Ref. [15].

The leading correction to the potential in Eq. (11) is independent of $r$ and thus does not contribute to energy level splittings. Therefore, from the absence of an $(r / L)^{2}$ term in (11), naively we might conclude that the impact of a depleted density of states on energy levels starts at $\mathcal{O}\left((r / L)^{3}\right)$. However, our calculation involved a sum over all momenta, and it is not immediately clear whether these corrections are produced primarily by small momenta,

\footnotetext{
${ }^{1}$ Note that, because the $\mathbf{n}$ lattice is not rotationally invariant, this choice of $\mathbf{r}$ is not fully generic. Since we do not know how the real depletion of states should be realized, we neglect angular dependence and merely use the finite-volume technique to extract the $|\mathbf{r}| / L$ scaling of the finite- $L$ corrections.
}

$q \ll 1 / r$ [i.e., $n \ll L /(2 \pi r)$ modes] or momenta $q \sim 1 / r$ [i.e., $n \sim L /(2 \pi r)$ modes]. As discussed in Sec. II, we are really interested in scale-dependent depletions, so we want to isolate the contributions of particular energy regimes to the $L$-dependence. We elect to focus on the highest energy scales that contribute significantly to the observable-in the present case, the scales $q \sim 1 / r$. Of course, higher energy states still contribute to the observables, and this is why precise atomic measurements can test weak-scale physics. Their effect on $L$-dependent corrections, however, is suppressed compared to $q \sim 1 / r$ states, generally leading to weaker constraints on the $L_{\text {eff }}$ (albeit at a different energy). We will demonstrate this point more explicitly in the following section.

We isolate the contribution of $q \sim 1 / r$ momenta by considering the difference between the continuum and discrete calculation in different cells in $\mathbf{n}=L \mathbf{q} /(2 \pi)$ space. We partition the continuum integration region in Eq. (6) into unit cubes corresponding to the terms in the discrete sum, such that the differences can be computed cell by cell. Since the integrand is even and the entire integration region is symmetric, we add the contributions from all eight cells that are related to each other by parity operations; the set of these eight cells is denoted by $P_{\mathbf{N}}$. For example, for the cell with the lowest vertex at $\mathbf{N}$, we find

$4 \pi^{2} L \Delta_{\mathbf{N}}=\sum_{\mathbf{M} \in P_{\mathbf{N}}}\left(\int_{\mathbf{M}} d^{3} n \frac{\cos \left(2 \pi n_{z} r / L\right)}{\mathbf{n}^{2}}-\frac{\cos \left(2 \pi M_{z} r / L\right)}{\mathbf{M}^{2}}\right)$,

where the integration in the first term is over the cell. Details of this calculation are given in Appendix B.

For $|\mathbf{N}| \gg 1$, the integrand is slowly varying over the unit cube, so the integral can be performed by doing a Taylor expansion around $\mathbf{N}$. In the regime of interest $N_{i} \sim L /(2 \pi r) \gg 1$, the result simplifies to

$$
\begin{aligned}
4 \pi^{2} L \Delta_{\mathbf{N}} \approx & \frac{4\left(11 N^{2}+9\right) c}{3 N^{6}} \\
& +\frac{(2 \pi r / L)\left(4 N^{2}\left(N_{z}-5\right)-6\left(4 N_{z}+5\right)\right) s}{3 N^{6}} \\
& +\frac{(2 \pi r / L)^{2}\left(2 N^{2}\left(N^{2}+N_{z}\right)-3\left(4 N_{z}+5\right)\right) c}{3 N^{6}} \\
& +\mathcal{O}\left((2 \pi r / L)^{3}\right),
\end{aligned}
$$

where $s$ and $c$ are the sine and cosine of $2 \pi N_{z} r / L$ and we presented only the first three terms in the $r / L$ expansion for brevity. There are $\mathcal{O}\left(N^{3}\right)$ such contributions, so the total finite-volume correction from $N_{i} \sim L /(2 \pi r)$ scales as

$$
\Delta_{\mathbf{N}} \times N^{3} \sim\left(\frac{1}{4 \pi^{2}}\right)\left(\frac{r}{L^{2}}+\frac{r^{2}}{L^{3}}+\frac{r^{3}}{L^{4}}+\ldots\right)
$$


Here, we have dropped numerical coefficients and taken the trigonometric functions to be $\mathcal{O}(1)$. Equation (14) is a somewhat surprising result because the term of order $1 / L^{2}$ is absent in the full result in Eq. (11). ${ }^{2}$ We have numerically confirmed this by computing the differences between continuum and discrete contributions to the potential from momenta of order $1 / r$. We conclude that the $1 / L^{2}$ corrections cancel if the depletion of states (effective box size) is energy-independent. If the depletion is instead energy-dependent, this cancellation might be spoiled. Since we do not know the precise functional form of the depletion, it is possible that the leading (additive) $r$-dependent corrections to the Coulomb potential scale as either $r / L^{2}$ or $r^{2} / L^{3}$. Below, we will consider both possibilities in estimating the sensitivity of atomic level splittings to the depletion scale.

\section{Finite-volume effects in hydrogen}

From the above, we are led to consider corrections to the Coulomb potential of the form

$$
\Delta V(r) \sim \frac{e^{2}}{L}\left(\frac{r}{L}\right)^{p}
$$

where $p$ may be 1 or 2 . The hydrogen wave functions are

$\psi_{n \ell m}=\frac{c_{n \ell}}{a^{3 / 2}}\left(\frac{2 r}{n a}\right)^{\ell} L_{n-\ell-1}^{2 \ell+1}\left(\frac{2 r}{n a}\right) e^{-r /(n a)} Y_{\ell}^{m}(\theta, \phi)$,

where $a=1 /\left(\alpha m_{e}\right), L_{p}^{q}(x)$ is an associated Laguerre polynomial and $c_{n \ell}$ is a normalization constant. The shifts to specific energy levels $\delta E_{n \ell}$ can be estimated as

$$
\begin{aligned}
\delta E_{n \ell} & =\langle n \ell m|\Delta V| n \ell m\rangle \\
& =c_{n \ell}^{2}\left(\frac{n}{2}\right)^{3} \int_{0}^{\infty} d u u^{2+2 \ell} \Delta V(\text { anu } / 2)\left[L_{n-\ell-1}^{2 \ell+1}(u)\right]^{2} e^{-u} .
\end{aligned}
$$

One of the most precisely measured splittings measured is the Lamb shift $\Delta E=E\left(2 S_{1 / 2}\right)-E\left(2 P_{1 / 2}\right)$. The experimental value is [17]

$$
\Delta E=1057845(9) \mathrm{kHz},
$$

in agreement with theoretical predictions (these depend on the proton radius, so the two inconsistent values give somewhat different predictions [18]). While there are slightly more precise experimental extractions of $\Delta E$ than Ref. [17], they rely on combinations multiple energy levels, complicating comparison with theory. For a direct

\footnotetext{
${ }^{2}$ Note that the $r$-independent Casimir term in Eq. (11) is absent from Eq. (14) because it is generated entirely by very low momenta, $q \sim 1 / L$, i.e., $|\mathbf{n}| \sim 1$.
}

measurement of $\Delta E$ like [17], the magnitude of the finite- $L$ correction is simply

$$
\delta(\Delta E)=\left|\delta E_{20}-\delta E_{21}\right| \sim \frac{e^{2}}{L}\left(\frac{a}{L}\right)^{p} .
$$

Demanding that this shift is less than the experimental uncertainty gives a lower bound on $L$ :

$$
L_{\text {eff }}(\mathrm{keV}) \gtrsim \begin{cases}10^{-3} \mathrm{~m} & p=1 \\ 10^{-6} \mathrm{~m} & p=2,\end{cases}
$$

where the characteristic energy is $\epsilon_{*} \sim \alpha m_{e} \sim \mathrm{keV}$. For comparison, the effective box size implied by the CKN DOS at this energy scale is

$$
L_{\mathrm{CKN}}(\mathrm{keV}) \sim 10^{14} \mathrm{~m} .
$$

\section{B. Anomalous magnetic moment of leptons}

The electron anomalous magnetic moment is one of the most precisely measured quantities in particle physics. Previous works have computed the corrections to lepton $g-2$ in a finite volume $[8,10,15,19]$. Since we are interested in energy-dependent effective volumes $L_{\text {eff }}(\epsilon)$, as above, we will need to modify these computations to isolate just the finite-volume terms that arise from a particular energy scale of the individual particles in the intermediate state. As before, we elect to focus on the energy scale which contributes the most to the observable, which for $g-2$ is $\sim m_{e}$. Unlike the leading-order Coulomb problem considered in the previous section, the intermediate states contributing to $g-2$ are multiparticle. This choice of energy scale has the additional advantage that all the intermediate state particles are (semi)relativistic and have energies of similar order. The incredible precision of $g-2$ measurements also means that the observable is sensitive to subdominant higher-energy intermediate states with the same property. We will argue at the end of this section that isolating these states instead leads to weaker constraints on $L_{\text {eff }}$ (albeit at a different energy scale).

The ordinary one-loop infinite-volume contribution to $g-2$ is [20]

$$
a=\frac{\alpha}{2 \pi} .
$$

We can rewrite the usual covariant Feynman integral in two useful ways. First is as a 3-momentum integral,

$$
a=6 \pi \alpha \int \frac{d^{3} k}{(2 \pi)^{3}} \frac{2 k\left(k-\sqrt{k^{2}+m_{e}^{2}}\right)+m_{e}^{2}}{3 k^{2} m_{e}^{2} \sqrt{k^{2}+m_{e}^{2}}} .
$$

Here, $k$ is the 3-momentum of an internal electron. (All results in this section can be repeated for the muon with the replacement $m_{e} \rightarrow m_{\mu}$.) The external electrons are at rest, 
and the external photon momentum is taken to zero. The integrand is dominated by $|k| \lesssim m_{e}$. In old-fashioned perturbation theory, where the intermediate states are on shell, the intermediate photon energy is of order $|k|$, and the electron energies are of order $\sqrt{k^{2}+m_{e}^{2}}$. Therefore, when $|k| \sim m_{e}$, all of the intermediate state particles have energies of order $m_{e}$. Thus, we can estimate a lower bound on the DOS at energies of order $m_{e}, g\left(m_{e}\right)$, by isolating the contribution to $g-2$ from $|k| \sim m_{e}$ and computing the effects of finite volume on it.

The second useful representation of the one-loop $g-2$ is the Schwinger parametrization,

$$
\begin{aligned}
a & =\frac{2 \sqrt{\pi} \alpha}{m_{e}^{2}} \int_{0}^{\infty} \frac{d \rho}{\sqrt{\rho}} \int \frac{d^{3} k}{(2 \pi)^{3}} f(\rho) e^{-\rho k^{2}} \\
& =\frac{\alpha}{4 \pi m_{e}^{2}} \int_{0}^{\infty} \frac{d \rho}{\rho^{2}} f(\rho) .
\end{aligned}
$$

Here, we have defined for convenience

$$
\begin{aligned}
f(\rho) & \equiv 4 m_{e}^{4} \rho^{2} \int_{0}^{1} d z z(1-z)^{2} e^{-\rho m_{e}^{2}(1-z)^{2}} \\
& =-2+m_{e} \sqrt{\pi \rho} \operatorname{Erf}\left(m_{e} \sqrt{\rho}\right)+2 e^{-m_{e}^{2} \rho} .
\end{aligned}
$$

Now, we compute the sensitivity of $a$ to the density of states $g(\epsilon)$ by placing the process in a finite box of size $L^{3}$. We discretize the momenta, $k \rightarrow 2 \pi n / L$, and perform the sum over the $\mathbf{n}$ lattice. In the Schwinger parametrization the result can be expressed in terms of theta functions,

$$
\sum_{n=-\infty}^{\infty} e^{-\rho(2 \pi n / L)^{2}}=\theta_{3}\left(e^{-4 \pi^{2} \rho / L^{2}}\right)=\frac{L}{2 \sqrt{\pi \rho}} \theta_{3}\left(e^{-L^{2} / 4 \rho}\right),
$$

where the two expressions are related by Poisson summation and $\theta_{3}(\tau)=\theta_{3}(0, \tau)$ in the definition of Eq. (8). In a finite box, the zero mode does not contribute to Feynman sums and must be omitted to avoid a spurious infrared divergence in the integral over $\rho$. Including this subtraction, we have

$$
\begin{aligned}
a^{L} & \equiv \frac{2 \sqrt{\pi} \alpha}{m_{e}^{2}} \int_{0}^{\infty} \frac{d \rho}{\sqrt{\rho}} f(\rho) L^{-3}\left(\theta_{3}\left(e^{-\frac{4 \pi^{2} \rho}{L^{2}}}\right)^{3}-1\right) \\
& =\frac{\alpha}{4 \pi m_{e}^{2}} \int_{0}^{\infty} \frac{d \rho}{\rho^{2}} f(\rho)\left(\theta_{3}\left(e^{-\frac{L^{2}}{4 \rho}}\right)^{3}-\frac{8 \pi^{\frac{3}{2}} \rho^{\frac{3}{2}}}{L^{3}}\right) .
\end{aligned}
$$

We can obtain an approximation to Eq. (27) as a series in $1 /\left(m_{e} L\right) \ll 1$. Details are given in Appendix A; here, we give only the result:
$a_{L} \approx \frac{\alpha}{2 \pi}\left(1-8.91\left(m_{e} L\right)^{-1}+35.65\left(m_{e} L\right)^{-2}-59.21\left(m_{e} L\right)^{-3}\right)$.

The leading correction, $-8.91\left(m_{e} L\right)^{-1}$, is similar in magnitude to the result obtained by Hasenfratz and Leutwyler [15] for the first corrections to the two-point function; the integrand in that case is equivalent to ours in the large- $\rho$ limit of $f(\rho)$. $^{3}$

The finite $L$ effects on the entire mode sum are of order $L^{-1}$. However, our interest is in the contribution to the finite $L$ effects from modes around $k \sim m_{e}$. In fact, it is easy to see that the $1 / L$ contribution in Eq. (28) comes entirely from the very low-momentum part of the mode sum, where $k \sim 1 / L$. Finite $L$ effects are equivalent to the error introduced by performing a Riemann sum $\mathbf{k} \rightarrow 2 \pi \mathbf{n} / L$ instead of an integral over $k$. The error contribution from the momentum bin at $\mathbf{N}$ (summed over all eight bins related by parity) is

$$
\Delta_{\mathbf{N}}=\frac{6 \pi \alpha}{L^{3}} \sum_{\mathbf{M} \in P_{\mathbf{N}}}\left[\int_{\mathbf{M}} d^{3} n g\left(\frac{2 \pi n}{m_{e} L}\right)-g\left(\frac{2 \pi M}{m_{e} L}\right)\right],
$$

where $g\left(k / m_{e}\right)$ is the dimensionless form of the integrand in Eq. (23). Repeating the Riemann error analysis of Sec. II A yields a total error estimate from the $\mathcal{O}\left(N^{3}\right)$ cells with $N \sim L m_{e} /(2 \pi) \gg 1$ (corresponding to $k \sim m_{e}$ )

$$
\Delta_{N} \times N^{3} \sim \frac{6 \pi \alpha}{\left(m_{e} L\right)^{2}} .
$$

We conclude that the $1 / L$ term in Eq. (28) arises from lowenergy modes, rather than typical modes of $k \sim m_{e}$. On the other hand, generic contributions from $k \sim m_{e}$ are expected to be $\mathcal{O}\left(1 / L^{2}\right)$ by the Riemann sum argument above. As before, the summation of all eight cells related by parity is key in obtaining the contribution of $k \sim m_{e}$ modes. These conclusions are readily verified numerically, performing sums over low and high momentum modes and comparing to integration over the same $k$-volumes. Thus, we will use the term of order $1 / L^{2}$ in Eq. (28) to estimate the effect on $g-2$ of depleting the density of states near $k \sim m_{e}$. This is in contrast to Ref. [10], which used $1 / L$ scaling; as we have just argued, however, such corrections are associated with low energies rather than $k \sim m_{e}$.

We are now in a position to convert these corrections into limits on the single-particle DOS at $\epsilon \sim m_{e}$, parametrized by an effective box size $L_{\mathrm{eff}}\left(m_{e}\right)$, and we can do the same for the muon. For our purposes, we will neglect existing

\footnotetext{
${ }^{3} f(\rho) \sim-2+\sqrt{\pi \rho m_{e}^{2}}$ at large $\rho$, up to exponentially small corrections. The appearance of $\sqrt{m_{e}^{2}}$ in this asymptotic expansion is responsible for the odd powers of $m_{e}$ appearing in Eq. (28), despite the fact that the integrand in Eq. (27) is an even function of $m_{e}$.
} 
discrepancies between experiment and theory. $a_{e}$ has been measured to about $1 \mathrm{ppb}$ and $a_{\mu}$ to about $0.3 \mathrm{ppm}$. Using the $1 / L^{2}$ scaling for the corrections, we find

$$
\begin{aligned}
& L_{\text {eff }}\left(m_{e}\right) \gtrsim 10^{5} m_{e}^{-1} \simeq 10 \mathrm{~nm} \\
& L_{\text {eff }}\left(m_{\mu}\right) \gtrsim 10^{4} m_{\mu}^{-1} \simeq 100 \mathrm{fm} .
\end{aligned}
$$

Note that we would obtain a much stronger bound if we discretized the state space by $1 / L$ at all scales; in this case, $a$ is corrected at $\mathcal{O}(1 / L)$. This is the standard scaling for finite-volume corrections to $g-2$ in a fixed box $[10,21]$ and was used in both Ref. [10] and a more recent analysis of the standard correlated UV-IR cutoff interpretation of CKN [8]. We are purposefully not using this scaling: as we have seen, most of the effects from a real finite box come from the lowest momenta of intermediate states, where the intermediate photon is much softer than $m_{e}$.

The effective sizes implied by CKN scaling are

$$
\begin{aligned}
& L_{\mathrm{CKN}}\left(m_{e}\right) \simeq 10^{21} m_{e}^{-1} \simeq 10^{5} \mathrm{~km} \\
& L_{\mathrm{CKN}}\left(m_{\mu}\right) \simeq 10^{19} m_{\mu}^{-1} \simeq 20 \mathrm{~km} .
\end{aligned}
$$

Clearly, we are in no danger of testing these densities.

In the discussion above, we focused on intermediate states with energy $m_{e}$ which generate the main contribution to $g-2$ and yield a constraint on $L\left(m_{e}\right)$. We could instead choose to focus on a higher energy scale $P \gg m_{e}$. One can still use Eq. (29) to estimate the effect of depletion of these states:

$$
\Delta_{N} \times N^{3} \sim a \frac{\left(m_{e} / P\right)^{2}}{(P L)^{2}} .
$$

Compared to Eq. (30), these corrections are suppressed by $\left(m_{e} / P\right)^{4} \ll 1$. For example, for $P \sim 100 \mathrm{GeV}$, we find much weaker constraints on $L_{\text {eff }}(P)$ for electron and muon $g-2$, respectively:

$$
\begin{aligned}
& L_{\text {eff }}(100 \mathrm{GeV}) \gtrsim 10^{-4} \mathrm{fm} \\
& L_{\text {eff }}(100 \mathrm{GeV}) \gtrsim 10^{-3} \mathrm{fm} .
\end{aligned}
$$

If $L$ is modeled as a power law in $1 / P$ with power less than or equal to 2 , then the strongest constraints on the normalization come from energies around the lepton mass, except in the case where the power is 2 and equally strong constraints are obtained from higher energy scales as well.

\section{Higher energy probes}

The finite-volume technique used above for the Lamb shift and $g-2$ could be applied to other precision processes. However, at much higher energies, observables are both predicted and measured with much lower precision. Instead, we make some simpler qualitative estimates.
Many detectors in high-energy physics have energy resolutions of order $0.1 \%-10 \%$. For example, the electromagnetic calorimeter at CMS is able to measure electron momenta to order $1 \%$ over a range $100 \mathrm{GeV}-1 \mathrm{TeV}$ [22]. Astrophysical observatories have access to even higher energies. For example, IceCube observes $\mathrm{PeV}$ neutrinos with an energy resolution of approximately 20\% [23], while the Pierre Auger Observatory measures approximately $100 \mathrm{EeV}$ cosmic rays with a 7\% resolution [24]. This implies an empirical bound on the effective length, $L_{\text {eff }}(100 \mathrm{GeV}) \gtrsim 1 \mathrm{fm}, \quad L_{\text {eff }}\left(10^{6} \mathrm{GeV}\right) \gtrsim 10^{-5} \mathrm{fm}, \quad$ and $L_{\text {eff }}\left(10^{11} \mathrm{GeV}\right) \gtrsim 10^{-10} \mathrm{fm}$. These length scales are factors of $10^{13}, 10^{9}$, and $10^{5}$ smaller than implied by the CKNinspired depletion of DOS in Eq. (3).

\section{HOT BOXES AND DEPLETED DOS}

Now, we return to the CKN bound, examining the relation between hot box thought experiments and the depleted DOS of Eq. (3) in more detail. We begin by generalizing Eq. (3) to a family of modified single-particle relativistic DOS,

$$
g(\epsilon)=\epsilon^{2} L_{\mathrm{eff}}(\epsilon)^{3}, \quad L_{\mathrm{eff}}(\epsilon)=\min \left(L, M_{p}^{-1}\left(M_{p} / \epsilon\right)^{n}\right)
$$

for some $n>1$. We ignore any $\mathcal{O}(1)$ numbers. It is also convenient to introduce the transition scale

$$
\hat{\epsilon} \equiv M_{p} /\left(M_{p} L\right)^{1 / n} .
$$

We take $n>1$ so that the transition energy satisfies $\hat{\epsilon} \gg$ $1 / L$ for all systems larger than the Planck length. For single-particle energies $\epsilon<\hat{\epsilon}$, the density of states is depleted by ordinary finite-volume effects related to the physical size of the system under consideration. For $\epsilon>\hat{\epsilon}$, it is depleted more strongly. We could further generalize the factors of $M_{p}$ in Eq. (35) to some lower scale associated with quantum gravity, if such a scale exists, but for simplicity, we will keep it as $M_{p}$.

Thus, we have a one-parameter family of models labeled by the depletion rate parameter $n$. For large $n$, the depletion rate as a function of energy is rapid, but the transition scale is also high. For small $n$, the depletion rate with energy is slow, but the transition scale is low. Primarily, we will be interested in $n \sim 1-2$, as we will see below.

Note that, since in any experimental system $L M_{p} \gg 1$, the transition between standard and depleted density occurs at scales far below $M_{p}$. We will also assume a cutoff on QFT at $E \sim M_{p}$.

Now, we estimate the effects of the DOS depletion on hot box gravitational backreaction, and the radiative correction to the cosmological constant, as a function of the depletion rate parameter $n$ in Eq. (35). 


\section{A. Hot boxes}

The energy and entropy of a thermal state of free particles are

$$
\begin{aligned}
E & =\int \operatorname{d\epsilon \epsilon g}(\epsilon) f_{ \pm}(\beta \epsilon) \\
S & =\int d \epsilon g(\epsilon)\left[\beta \epsilon f_{ \pm}(\beta \epsilon) \pm \log \left(1 \pm e^{-\beta \epsilon}\right)\right] \\
& =-\int \operatorname{deg}(\epsilon)\left[f_{ \pm} \ln f_{ \pm} \pm\left(1 \mp f_{ \pm}\right) \ln \left(1 \mp f_{ \pm}\right)\right],
\end{aligned}
$$

where $f_{ \pm}$is the Fermi-Dirac (+) or Bose-Einstein (-) distribution function and $\beta=1 / T$. We will estimate the energy and the entropy for the depleted density of states in Eq. (35).

First we consider the Fermi-Dirac case. For high but subPlanckian temperatures $M_{p} \gg T \gg \hat{\epsilon}$, the energy scales as

$$
\begin{aligned}
E & =\int^{\hat{\epsilon}} d \epsilon \frac{\epsilon^{3} L^{3}}{e^{\beta \epsilon}+1}+\int_{\hat{\epsilon}} d \epsilon \frac{\left(M_{p} / \epsilon\right)^{3 n-3}}{e^{\beta \epsilon}+1} \\
& \sim M_{p} \begin{cases}\left(T / M_{p}\right)^{4-3 n} & 1<n<4 / 3 \\
\ln \left[(L T) /\left(L M_{p}\right)^{1 / 4}\right] & n=4 / 3 \\
\left(L M_{p}\right)^{3-4 / n} & n>4 / 3,\end{cases}
\end{aligned}
$$

where we drop $\mathcal{O}(1)$ factors and take $n>1$ for reasons described above. For $n>4 / 3$, the energy is dominated by contributions from modes near $\hat{\epsilon}$. As in CKN, we can compare the Schwarzschild scale $E / M_{p}^{2}$ to the system size $L$. The bound $L>E / M_{p}^{2}$ is satisfied for $n<2$ and saturated for $n=2$. For the entropy, the scaling behavior is

$$
S \sim\left(L M_{p}\right)^{3-3 / n} .
$$

In the Bose-Einstein case, at high $M_{p} \gg T \gg \hat{\epsilon}$, the energy behaves as

$E=\int^{\hat{\epsilon}} d \epsilon \frac{\epsilon^{3} L^{3}}{e^{\beta \epsilon}-1}+\int_{\hat{\epsilon}} d \epsilon \frac{\left(M_{p} / \epsilon\right)^{3 n-3}}{e^{\beta \epsilon}-1} \sim T\left(L M_{p}\right)^{3-3 / n}$.

again dropping $\mathcal{O}(1)$ factors. This scaling applies for $n>1$ and is different from the Fermi-Dirac case due to large occupation numbers in low-energy modes. Comparing the Schwarzschild scale $E / M_{p}^{2}$ to the system size $L$, we find that black holes are not formed for any $T<M_{p}$ if $n \leq 3 / 2$. For $n>3 / 2$, however, there is still a bound on the temperatures,

$$
T<\frac{M_{p}}{\left(L M_{p}\right)^{2-3 / n}}
$$

In fact, for $n=2$, the bound on $T$ obtained in this manner is approximately the same as in the original CKN bound. So, in this case, the depletion of the density of states does not automatically eliminate black holes at high temperatures in finite-size bosonic systems.

On the other hand, no depleted single-particle DOS nor correlated UV-IR cutoff on Feynman integrals can remove black holes formed by nonthermal states of soft bosonic modes with enormous occupation numbers. Some states must be removed in a way that depends on the occupancy. Instead, however, we can ask whether the entropy is reduced in a way consistent with the CKN bound. The entropy is

$$
S \sim\left(L M_{p}\right)^{3-3 / n} \log (\beta \hat{\epsilon}) .
$$

Up to the logarithm, it saturates the $L^{3 / 2}$ scaling for $n=2$.

From these thought experiments, we conclude that the range $1<n \leq 2$ is of interest, with possibly special roles played by $n=2$ and $n=3 / 2 . n=2$, the model used for comparison in Sec. II, is the more conservative of the two; it corresponds a faster rate of depletion, but it turns on at higher energies, corresponding to a lesser overall depletion at any given scale.

\section{B. Cosmological constant}

Finally, let us consider the impact of a depleted DOS on the cosmological constant. In the power-law models above, the QFT vacuum energy contribution to the $\mathrm{cc}$ is finite if $n>4 / 3$ and scales as

$$
\delta \rho \sim H^{3} \int \operatorname{d\epsilon \epsilon } g(\epsilon) \sim \hat{\epsilon}^{4},
$$

where $H$ is the Hubble scale. Unlike the local observables considered in Sec. II, the cc does not have a preferred energy scale $\epsilon_{*}$; rather, $\delta \rho$ is dominated by the most numerous modes around $\hat{\epsilon}$.

The relative size of the correction in Eq. (43) is

$$
\frac{\delta \rho}{\rho} \sim\left(\frac{\rho}{M_{p}^{4}}\right)^{2 / n-1} .
$$

We see that for $n \leq 2$, the cc appears to be technically natural, i.e., the quantum correction is at most of the size of the cc itself. While $n>2$ still gives rise to a finite $\delta \rho$, the correction exceeds the observed cc because the transition scale $\hat{\epsilon}$ becomes too large. For $n=2$, $\hat{\epsilon} \sim \mathrm{meV}$. We emphasize that we do not interpret this as a transition energy beyond which QFT breaks down in all experiments. Rather, $\epsilon>\hat{\epsilon}$ merely marks the energy scale at which the characteristic momentum spacing between independent field modes exceeds $H \sim 10^{-33} \mathrm{eV}$. Systems much smaller 
than the horizon size (i.e., laboratory experiments) are sensitive to a different DOS (i.e., different $L$ and $\hat{\epsilon}$ ) and therefore cannot constrain fluctuations over approximately $1 / H$, as emphasized by CKN [1]. This point of view is in contrast with the idea that laboratory experiments which measure the gravitation of vacuum fluctuations can be used to justify the standard (UV-sensitive) QFT estimate of the vacuum energy [25]. In fact, the $(g-2)$ calculation in Sec. II B shows that even some of the most precise probes of vacuum fluctuations are not sensitive to the depletion of the DOS needed to address the cc fine-tuning problem.

\section{CONCLUSION}

Quantum field theory provides an accurate description of local observables over an enormous range of energy scales, including high-energy collider processes and precision measurements in atomic physics. However, a naive application of QFT to extensive systems runs into conceptual problems, including the violation of entropy bounds or strong gravitational backreaction even for rather mundane energy densities and volumes. For example, a $10 \mathrm{~m}$ room filled with $T \sim 10 \mathrm{GeV}$ plasma is within its own Schwarzschild radius, implying that nongravitational QFT must break down. Based on these thought experiments, CKN proposed a correlation between the UV and the IR cutoffs on QFT [1]. We have explored an alternative interpretation proposed in Ref. [10], in which the independent degrees of freedom are depleted in an energy scaledependent way. An analogous depletion has been suggested in partonic models of holography [6]. We studied a simple phenomenological implementation of a state depletion, motivated by the "hot box" thought experiments of CKN.

For local observables, the DOS depletion can be viewed as a finite-volume effect, where the volume depends on the characteristic energy scale of the observable. We have applied this reasoning to estimate the corrections to two sensitive probes of QFT, lepton $g-2$ and the hydrogen Lamb shift, finding that, despite their incredible precision, these measurements are far from being sensitive to the depletions motivated by quantum gravity. Tests of the DOS are, however, a novel axis for testing QFT. It would be interesting explore other observations that probe the DOS at different energy scales, such as high-energy cosmic-ray scattering.

An interesting application of these DOS models is the calculation of the QFT vacuum energy contribution to the cosmological constant, which becomes UV insensitive if the depletion is rapid enough. For a DOS that saturates the $\mathrm{CKN}$ bound, we find that the $\mathrm{cc}$ is technically natural.

The most important questions, unaddressed here, are how Lorentz invariance emerges and whether the QFT entropy scaling $S_{\mathrm{QFT}} \sim\left(M_{p} L\right)^{3 / 2}$ can be motivated by microscopic models analogous to the partonic models of holography. The Holographic Space Time models of Banks and Fischler [26-28], for example, have been argued to realize just such a scaling [29].

\section{ACKNOWLEDGMENTS}

We thank Tom Banks, Ben Lillard, Aleksey Cherman, and Theo Jacobson for useful discussions. P. D. acknowledges support from the U.S. Department of Energy under Grant No. DE-SC0015655. This manuscript has been authored by Fermi Research Alliance, LLC, under Contract No. DE-AC02-07CH11359 with the U.S. Department of Energy, Office of Science, Office of High Energy Physics.

\section{APPENDIX A: ANALYTIC RESULTS FOR FINITE-VOLUME CORRECTIONS}

In this Appendix, we give details of the calculation of the $1 / L$ series for the Coulomb problem and lepton magnetic moments, Eqs. (11) and (28), respectively.

\section{Coulomb potential}

We begin by splitting the $\rho$ integration in the first line of Eq. (11) into intervals $[0, \hat{\rho}]$ and $(\hat{\rho}, \infty)$, where $\hat{\rho}$ is chosen such that $4 \pi^{2} \hat{\rho} / L^{2} \ll 1$. In the first interval, we can expand the integrand in $4 \pi^{2} \rho / L^{2} \ll 1$, perform the integration, and then expand the result in $r / L$ :

$$
\begin{aligned}
I_{1} & =-e^{2} \int_{0}^{\hat{\rho}} d \rho \frac{1}{L^{3}}\left[\theta_{3}\left(0, e^{-4 \pi^{2} \rho / L^{2}}\right) \theta_{3}\left(0, e^{-4 \pi^{2} \rho / L^{2}}\right) \theta_{3}\left(-\frac{r}{L}, e^{-4 \pi^{2} \rho / L^{2}}\right)-1\right] \\
& \approx-\frac{e^{2}}{4 \pi r}\left[1-\frac{r}{L}\left(4 \pi \hat{\rho} / L^{2}+L / \sqrt{\pi \hat{\rho}}\right)+\frac{r^{3}}{12 \sqrt{\pi} \hat{\rho}^{3 / 2}}\right] .
\end{aligned}
$$

The final expansion in $r / L$ is valid if $r / L \ll \sqrt{\hat{\rho} / L^{2}}$. In the second interval, $(\hat{\rho}, \infty)$, the integrand can be expanded in $r / L$ because the function is analytic once the singularity at $\rho=0$ is excluded. The result is

$$
I_{2}=-e^{2} \int_{\hat{\rho}}^{\infty} d \rho \frac{1}{L^{3}}\left[\theta_{3}\left(0, e^{-4 \pi^{2} \rho / L^{2}}\right) \theta_{3}\left(0, e^{-4 \pi^{2} \rho / L^{2}}\right) \theta_{3}\left(-\frac{r}{L}, e^{-4 \pi^{2} \rho / L^{2}}\right)-1\right]
$$




$$
\approx-\frac{e^{2}}{4 \pi r} \sum_{k=0} c_{2 k+1}(r / L)^{2 k+1}
$$

where the first two coefficients are given by

$$
\begin{aligned}
& c_{1}=\int_{\hat{\rho}}^{\infty} d \rho\left[\theta_{3}\left(0, e^{-4 \pi^{2} \rho / L^{2}}\right)^{3}-1\right] \\
& c_{3}=\int_{\hat{\rho}}^{\infty} d \rho \frac{\pi^{2}}{2} \theta_{3}\left(0, e^{-4 \pi^{2} \rho / L^{2}}\right)^{2} \theta_{3}^{\prime}\left(0, e^{-4 \pi^{2} \rho / L^{2}}\right) .
\end{aligned}
$$

While $I_{1}$ and $I_{2}$ individually depend on the unphysical parameter $\hat{\rho}$, their sum does not, which we checked for a large range of $\hat{\rho} / L^{2}$. The resulting $r / L$ expansion of $V_{R}=$ $I_{1}+I_{2}$ agrees with the one given in Eq. (11) obtained by a fully numerical method.

\section{Anomalous magnetic moment}

In this Appendix, we derive the $1 / L$ expansion for the finite-volume corrections to $g-2$ given in Eq. (28). To simplify notation, we define

$$
y \equiv m_{e} L .
$$

Let $t=\frac{L^{2}}{4 \pi \rho}$. In terms of $t$, we have

$$
a_{L}=\frac{\alpha}{y^{2}} \int_{0}^{\infty} d t t^{-3 / 2} f\left(\frac{L^{2}}{4 \pi t}\right)\left(\theta_{3}\left(e^{-\pi / t}\right)^{3}-1\right)
$$

Now, we split the integration into two regimes: $0 \leq t \leq y$, and $y<t<\infty$. In these regimes, different parts of the integrands can be replaced by asymptotic expansions, up to terms exponentially small in $y$ :

$$
\begin{aligned}
& a_{L}=\frac{\alpha}{2 \pi}\left(\mathcal{I}_{1}+\mathcal{I}_{2}\right) \\
& \mathcal{I}_{1} \approx 2 \pi \int_{0}^{y} d t\left(-\frac{2}{y^{2} t^{\frac{3}{2}}}+\frac{1}{2 y t^{2}}\right)\left(\theta_{3}\left(e^{-\pi / t}\right)^{3}-1\right) \\
& \mathcal{I}_{2} \approx 2 \pi \int_{y}^{\infty} d t\left(-\frac{2}{y^{2} t^{\frac{3}{2}}}+\frac{2}{y^{2} t^{\frac{3}{2}}} e^{-\frac{y^{2}}{4 t}}+\frac{1}{2 y t^{2}} \operatorname{Erf}\left(\frac{y}{2 \sqrt{\pi t}}\right)\right)\left(t^{3 / 2}-1\right) .
\end{aligned}
$$

First, consider $\mathcal{I}_{1}$. We can further split the integration range into $0 \leq t \leq \hat{t}$ and $\hat{t}<t<y$, for some $1 \ll \hat{t} \ll y$. $\hat{t}$ is arbitrary as long as it falls in this range. Then, we can write

$$
\begin{aligned}
& \mathcal{I}_{1}=\frac{c_{1}(\hat{t})}{y}+\frac{c_{2}(\hat{t})}{y^{2}}+2 \pi \int_{\hat{t}}^{y} d t\left(-\frac{2}{y^{2} t^{\frac{3}{2}}}+\frac{1}{2 y t^{2}}\right)\left(t^{\frac{3}{2}}-1\right) \\
& c_{1}=2 \pi \int_{0}^{\hat{t}} d t\left(\frac{1}{2 t^{2}}\right)\left(\theta_{3}\left(e^{-\pi / t}\right)^{3}-1\right) \\
& c_{2}=2 \pi \int_{0}^{\hat{t}} d t\left(-\frac{2}{t^{\frac{3}{2}}}\right)\left(\theta_{3}\left(e^{-\pi / t}\right)^{3}-1\right),
\end{aligned}
$$

where $c_{1}$ and $c_{2}$ can be evaluated numerically for some arbitrary choice of $\hat{t}$, and the remaining integral can be evaluated analytically. We find

$$
\mathcal{I}_{1}=2 \pi\left(y^{-1 / 2}-3.42 y^{-1}+6.17 y^{-2}-4 y^{-5 / 2}\right) .
$$

$\mathcal{I}_{2}$ can be treated similarly. It is convenient to redefine $\tilde{t}=t / y^{2}$ so that

$$
\mathcal{I}_{2}=2 \pi \int_{1 / y}^{\infty} d \tilde{t}\left(-2+2 e^{-\frac{1}{4 \pi \tilde{t}}}+\frac{1}{2 \sqrt{\tilde{t}}} \operatorname{Erf}\left(\frac{1}{2 \sqrt{\pi \tilde{t}}}\right)\right)\left(1-\frac{1}{\tilde{t}^{3 / 2} y^{3}}\right)
$$

and further split the integration range into $1 / y \leq \tilde{t} \leq \hat{\tilde{t}}$ and $\hat{\tilde{t}}<\tilde{t}<\infty$, for some $1 / y \ll \hat{\tilde{t}} \ll 1$. As before, $\hat{\tilde{t}}$ is arbitrary as long as it falls in this range. Then, 


$$
\begin{aligned}
& \mathcal{I}_{2}=c_{0}(\hat{\tilde{t}})+\frac{c_{3}(\hat{\tilde{t}})}{y^{3}}+2 \pi \int_{1 / y}^{\hat{\tilde{t}}} d \tilde{t}\left(-2+\frac{1}{2 \sqrt{\tilde{t}}}\right)\left(1-\frac{1}{\tilde{t}^{3 / 2} y^{3}}\right) \\
& c_{0}=2 \pi \int_{\hat{\tilde{t}}}^{\infty} d \tilde{t}\left(-2+2 e^{-\frac{1}{4 \pi \tilde{t}}}+\frac{1}{2 \sqrt{\tilde{t}}} \operatorname{Erf}\left(\frac{1}{2 \sqrt{\pi \tilde{t}}}\right)\right) \\
& c_{3}=2 \pi \int_{\hat{\tilde{t}}}^{\infty} d \tilde{t}\left(-2+2 e^{-\frac{1}{4 \pi \tilde{t}}}+\frac{1}{2 \sqrt{\tilde{t}}} \operatorname{Erf}\left(\frac{1}{2 \sqrt{\pi \tilde{t}}}\right)\right)\left(-\frac{1}{\tilde{t}^{3 / 2}}\right),
\end{aligned}
$$

where, as before, $c_{0}$ and $c_{3}$ can be evaluated numerically for some arbitrary choice of $\hat{\tilde{t}}$, and the integral can be evaluated analytically. We find

$$
\mathcal{I}_{2}=2 \pi\left(0.159-y^{-1 / 2}+2 y^{-1}+\frac{1}{2} y^{-2}+4 y^{-5 / 2}-9.42 y^{-3}\right)
$$

All the fractional powers of $y$ cancel between $I_{1}$ and $I_{2}$. In total, and restoring $y \rightarrow m_{e} L$,

$$
a_{L} \approx \frac{\alpha}{2 \pi}\left(1-8.91\left(m_{e} L\right)^{-1}+35.65\left(m_{e} L\right)^{-2}-59.21\left(m_{e} L\right)^{-3}\right) .
$$

\section{APPENDIX B: FINITE-VOLUME EFFECTS FROM RIEMANN SUM ERROR}

In this Appendix, we derive the scaling of finite-box corrections to the Coulomb potential as a function of the exchanged photon's energy scale by directly computing the difference between continuum and discretized versions in each momentum cell.

We define the complete continuum potential integral as

$$
f(r)=\frac{1}{4 \pi^{2} L} \int d^{3} n \frac{\cos \left(2 \pi n_{z} r / L\right)}{\mathbf{n}^{2}}
$$

where we changed variables to the dimensionless momentum $\mathbf{n}=\mathbf{q} L /(2 \pi)$; we have not made use of the three parity symmetries yet, so each integral is over $(-\infty,+\infty)$. The corresponding discrete sum is

$$
\hat{f}(r)=\frac{1}{4 \pi^{2} L} \sum_{\mathbf{N}}^{\prime} \frac{\cos \left(2 \pi N_{z} r / L\right)}{\mathbf{N}^{2}}
$$

where we used capital $N_{i}$ to distinguish the summation indices from the integration variables $n_{i}$; the prime denotes omission of $\mathbf{N}=\mathbf{0}$. We define the difference

$$
\Delta=f(r)-\hat{f}(r)
$$

This is most easily computed if we divide up the $d^{3} n$ integration region in Eq. (B1) into unit cubes corresponding to each term in the sum Eq. (B2):

$$
f(r)=\frac{1}{4 \pi^{2} L} \sum_{\mathbf{N}} \int_{\mathbf{N}} d^{3} n \frac{\cos \left(2 \pi n_{z} r / L\right)}{\mathbf{n}^{2}}
$$

where each cube has its lowest vertex at $\mathbf{N}$.

There are eight boxes/integration regions that have $\mathbf{0}$ at a vertex of their boundary, so we treat these separately; these eight cubes have starting vertices at

$$
\begin{aligned}
& (-1,-1,-1), \quad(-1,-1,0), \quad(-1,0,-1), \quad(0,-1,-1) \\
& (0,0,0), \quad(0,0,-1), \quad(0,-1,0), \quad(-1,0,0) . \quad(B 5)
\end{aligned}
$$

Since in this eight box region $n_{z} \leq 1$ and we are interested in $r / L \ll 1$, we can expand the cosine in the integrand and add up the numerically evaluated contributions of all eight boxes, yielding

$$
4 \pi^{2} L \Delta_{|\mathbf{N}| \leq 1} \approx 10.5-\frac{5}{2}\left(\frac{2 \pi r}{L}\right)^{2}
$$

where 10.5 and $5 / 2$ result from doing $d^{3} n$ integrals numerically. The same argument holds for other regions with $|\mathbf{N}| \sim 1$ - they contribute at $\mathcal{O}(1 / L)$ and $\mathcal{O}\left(r^{2} / L^{3}\right)$ to $\Delta$, since there are $\mathcal{O}(1)$ such terms.

For $|\mathbf{N}| \gg 1$, the integrands in each term in $\Delta$ are slowly varying, so we can perform a Taylor expansion in each box, $\mathbf{N}$ :

$$
\begin{aligned}
& \frac{\cos \left(2 \pi n_{z} r / L\right)}{\mathbf{n}^{2}}-\frac{\cos \left(2 \pi N_{z} r / L\right)}{\mathbf{N}^{2}} \\
& \approx \nabla_{i} g(\mathbf{n}-\mathbf{N})_{i}+\frac{1}{2} \nabla_{i} \nabla_{j} g(\mathbf{n}-\mathbf{N})_{j}(\mathbf{n}-\mathbf{N})_{j}+\ldots
\end{aligned}
$$


where $g$ is a shorthand for $\cos \left(2 \pi n_{z} r / L\right) / \mathbf{n}^{2}$; the derivatives can be evaluated, while the $d^{3} n$ give

$$
\begin{aligned}
\int_{n_{x}}^{n_{x}+1} \int_{n_{y}}^{n_{y}+1} \int_{n_{y}}^{n_{y}+1} d^{3} n(\mathbf{n}-\mathbf{N}) & =\frac{1}{2}(1,1,1) \\
\int_{n_{x}}^{n_{x}+1} \int_{n_{y}}^{n_{y}+1} \int_{n_{y}}^{n_{y}+1} d^{3} n(\mathbf{n}-\mathbf{N}) \otimes(\mathbf{n}-\mathbf{N}) & =\left(\begin{array}{lll}
1 / 3 & 1 / 4 & 1 / 4 \\
1 / 4 & 1 / 3 & 1 / 4 \\
1 / 4 & 1 / 4 & 1 / 3
\end{array}\right) .
\end{aligned}
$$

We must add all regions related by parity to exhibit the symmetries of the whole integration region and integrand. There are three parity transformations that can be used individually, or combined, such that a single region is related to seven others by these transformations; parity in the $i$ th direction maps $-N_{i}-1 \rightarrow+N_{i}$. Therefore, if we start with a box with its lowest vertex at $\left(N_{x}, N_{y}, N_{z}\right)$, we relate it to seven others by these parity operations. We denote the whole set of boxes generated in this way by $P_{\mathrm{N}}$ :

$$
\begin{aligned}
P_{\mathbf{N}}= & \left\{\left(-N_{x}-1,-N_{y}-1,-N_{z}-1\right),\left(N_{x},-N_{y}-1,-N_{z}-1\right),\right. \\
& \left(N_{x}, N_{y},-N_{z}-1\right),\left(N_{x}, N_{y}, N_{z}\right), \\
& \left(-N_{x}-1, N_{y},-N_{z}-1\right),\left(-N_{x}-1, N_{y}, N_{z}\right) \\
& \left.\left(-N_{x}-1,-N_{y}-1, N_{z}\right),\left(N_{x},-N_{y}-1, N_{z}\right)\right\} .
\end{aligned}
$$

For example, taking $\mathbf{N}=\mathbf{0}$ gives the set of eight boxes surrounding the origin in Eq. (B5). Adding up the contributions of all these boxes for $N \gg 1$ and $N_{z} \gg 1$ yields an error of

$$
4 \pi^{2} L \Delta_{N \gg 1, N_{z} \gg 1} \approx \frac{12}{N^{4}} \cos N_{z} \epsilon+\frac{4\left(2-N^{2} \epsilon^{2}\right)}{3 N^{4}} \cos N_{z} \epsilon+\frac{16 N_{z} \epsilon}{3 N^{4}} \sin N_{z} \epsilon,
$$

where $\epsilon=2 \pi r / L$ and the first (second and third) term arises from the first (second) term in the Taylor expansion in Eq. (B7). In the regime $N \sim N_{z} \gg 1$, there are $\mathcal{O}\left(N^{3}\right)$ boxes that contribute [approximately $4 \pi N^{2} d N$ with $d N \sim \mathcal{O}(N)$ ]; the total contribution to the Riemann sum error is therefore of order $N^{3}$ times Eq. (B10). For $N_{i} \sim L / r \sim 1 / \epsilon$, the trigonometric functions are $\mathcal{O}(1)$, and we obtain the schematic scaling of $1 / L$ corrections given in Eq. (14).

An analogous calculation can be performed for the $g-2$ integral in Eq. (23). The result is given in Eq. (30).

[1] A. G. Cohen, D. B. Kaplan, and A. E. Nelson, Effective Field Theory, Black Holes, and the Cosmological Constant, Phys. Rev. Lett. 82, 4971 (1999).

[2] T. Banks, On the limits of effective quantum field theory: Eternal inflation, landscapes, and other mythical beasts, arXiv:1910.12817.

[3] C.B. Thorn, Reformulating string theory with the $1 / N$ expansion, The First International A.D. Sakharov Conference on Physics (Nova Science Publishers, New York, 1991).

[4] G. 't Hooft, Dimensional reduction in quantum gravity, Conf. Proc. C 930308, 284 (1993), arXiv:gr-qc/9310026.

[5] L. Susskind, Strings, black holes and Lorentz contraction, Phys. Rev. D 49, 6606 (1994).

[6] L. Susskind, The World as a hologram, J. Math. Phys. (N.Y.) 36, 6377 (1995).

[7] J. Bramante and E. Gould, Material matter effects in gravitational UV/IR mixing, Phys. Rev. D 101, 084022 (2020).
[8] A. G. Cohen and D. B. Kaplan, Gravitational contributions to the electron $g$-factor, arXiv:2103.04509.

[9] H. Davoudiasl, Cohen-Kaplan-Nelson bound and minimum coupling in effective field theories, arXiv:2105 .01116 .

[10] T. Banks and P. Draper, Remarks on the Cohen-KaplanNelson bound, Phys. Rev. D 101, 126010 (2020).

[11] T. Banks, SUSY breaking, cosmology, vacuum selection and the cosmological constant in string theory, in ITP Workshop on SUSY Phenomena and SUSY GUTS (1995), https://inspirehep.net/conferences/970017?ui-citationsummary=true.

[12] T. Banks, W. Fischler, S. H. Shenker, and L. Susskind, M theory as a matrix model: A Conjecture, Phys. Rev. D 55, 5112 (1997).

[13] K. R. Dienes, Modular invariance, finiteness, and misaligned supersymmetry: New constraints on the numbers of physical string states, Nucl. Phys. B429, 533 (1994). 
[14] A. Cherman, M. Shifman, and M. Ünsal, Bose-Fermi cancellations without supersymmetry, Phys. Rev. D 99, 105001 (2019).

[15] P. Hasenfratz and H. Leutwyler, Goldstone boson related finite size effects in field theory and critical phenomena with $\mathrm{O}(N)$ symmetry, Nucl. Phys. B343, 241 (1990).

[16] A. S. Kronfeld, Uses of effective field theory in lattice QCD: Chapter 39 in at the frontiers of particle physics, handbook of QCD, arXiv:hep-lat/0205021.

[17] S. R. Lundeen and F. M. Pipkin, Measurement of the Lamb Shift in Hydrogen, $n=2$, Phys. Rev. Lett. 46, 232 (1981).

[18] M. I. Eides, H. Grotch, and V. A. Shelyuto, Theory of Light Hydrogenic Bound States (Springer-Verlag, Berlin, 2007), Vol. 222.

[19] Z. Davoudi, J. Harrison, A. Jüttner, A. Portelli, and M. J. Savage, Theoretical aspects of quantum electrodynamics in a finite volume with periodic boundary conditions, Phys. Rev. D 99, 034510 (2019).

[20] J. S. Schwinger, On Quantum electrodynamics and the magnetic moment of the electron, Phys. Rev. 73, 416 (1948).

[21] Z. Davoudi and M. J. Savage, Finite-volume electromagnetic corrections to the masses of mesons, baryons and nuclei, Phys. Rev. D 90, 054503 (2014).
[22] A. M. Sirunyan et al. (CMS Collaboration), Search for highmass resonances in dilepton final states in proton-proton collisions at $\sqrt{s}=13 \mathrm{TeV}$, J. High Energy Phys. 06 (2018) 120.

[23] M. G. Aartsen et al. (IceCube Collaboration), Probing the origin of cosmic rays with extremely high energy neutrinos using the IceCube observatory, Phys. Rev. D 88, 112008 (2013).

[24] A. Aab et al. (Pierre Auger Collaboration), Measurement of the cosmic-ray energy spectrum above $2.5 \times 10^{18} \mathrm{eV}$ using the pierre auger observatory, Phys. Rev. D 102, 062005 (2020).

[25] J. Polchinski, The cosmological constant and the string landscape, in 23rd Solvay Conference in Physics: The Quantum Structure of Space and Time (World Scientific Publishing, Singapore, 2006).

[26] T. Banks and W. Fischler, Holographic theory of accelerated observers, the S-matrix, and the emergence of effective field theory, arXiv:1301.5924.

[27] T. Banks and W. Fischler, Cosmological implications of the Bekenstein bound, arXiv:1810.01671.

[28] T. Banks and W. Fischler, Holographic space-time, Newton's Law, and the dynamics of horizons, arXiv:2003.03637.

[29] T. Banks (private communication). 